\title{
A fully automated high-throughput plasmid purification workstation for the generation of mammalian cell expression-quality DNA
}

\author{
Michael Cohen, David E. Randolph, Maria E. Lozano, Paul W. Anderson, John Crissman, \\ Franz J. Triana, Thomas Cujec*
}

Department of Biologics Automation and High-Throughput Technologies, Eli Lilly and Company Biotechnology Center, San Diego, CA, USA

\begin{abstract}
A B S T R A C T

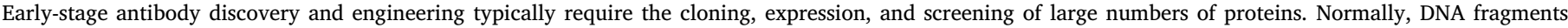

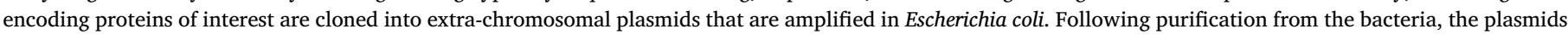

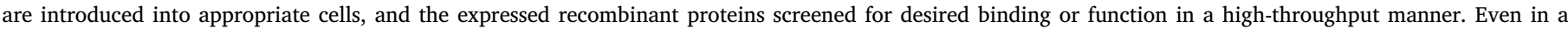

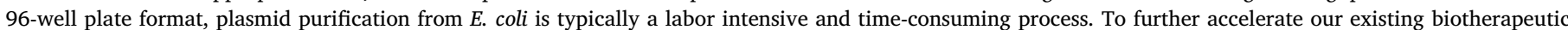

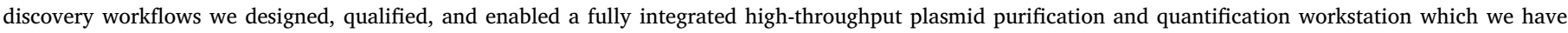

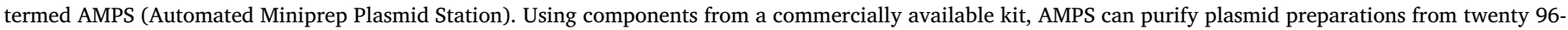

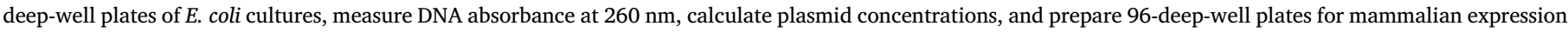

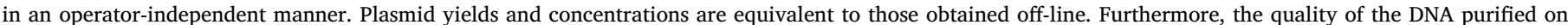

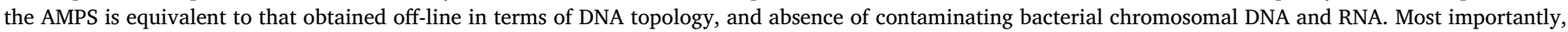

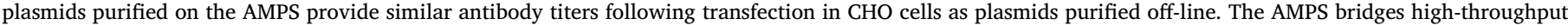

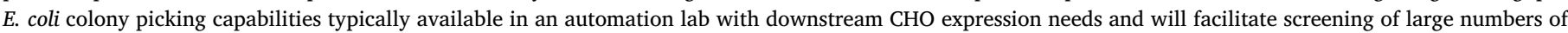
biotherapeutics in binding and cell assay screens.
\end{abstract}

\section{Introduction}

Protein therapeutics are amino acid-based drugs that are often genetically engineered and synthetically produced. In 2018, the market value of antibody-based therapeutics exceeded $\$ 100$ billion with sales forecast to reach $\$ 300$ billion by 2025 [1-3]. The robust growth in annual sales reflects the important role this class of therapeutics plays in treating a wide variety of diseases.

Antibodies are the most common class of commercial biotherapeutics. Antibodies are Y-shaped proteins formed by two light chain (LC) and heavy chain (HC) dimers linked together by disulfide bonds. They are produced in vertebrates as part of the adaptive immune response to help the organism clear foreign agents. The antibody LC and HC each contain variable light and heavy domains (VL and $\mathrm{VH}$, respectively), as well as constant regions. After activation of the adaptive immune response, a vast array of diverse sequences in the VL and VH domains are generated by a variety of mechanisms including recombination of germline sequences, deletion, and addition of nucleotides in specific segments of the variable regions, as well as by somatic hypermutation. B-cells producing antibodies that bind specifically and with high affinity to the foreign protein are selectively amplified [4-6]. In the context of antibody discovery, animals are often immunized with a target protein and a wide variety of diverse antibodies are generated as part of the im- mune repertoire [2]. Automation resources are necessary to support the high-throughput methods designed to recover antibodies generated by the animal immune system in a recombinant form and to identify those having the desired binding profile and functional activity.

Numerous factors must be considered in the development of a successful antibody therapeutic. The antibody must bind to the target of interest with high selectivity at the correct location on the target, and with optimal affinity to elicit the desired functional biological activity. Antibody expression levels, as well as biochemical and biophysical characteristics are important for the successful development of a manufacturable product, moreover low immunogenicity and low non-specific binding to host proteins play an important role in efficacy and drug disposition [7-10].

Typically, discovery campaigns involve the cloning, expression, and screening of large numbers of antibodies to find molecules having the desired binding profile and functional activity. Lead candidates are then often "engineered" by introducing amino acid changes at different positions in the protein and the variants are expressed and re-screened to find optimized molecules that balance the many characteristics required for a successful therapeutic [11-13].

In a typical high-throughput antibody discovery workflow, DNA fragments encoding the VL and VH domains of an antibody are cloned into plasmids upstream of the antibody constant sequences (kappa or

\footnotetext{
* Corresponding author.

E-mail address: cujecth@lilly.com (T. Cujec).
} 
Fc) and under control of the appropriate regulatory elements for expression in the desired host cells. Numerous high-throughput cloning strategies have been developed to clone 100-1000s of antibody variable domains at a time [14-19]. Following transformation of the recombinant plasmids into Escherichia coli, multiple extra-chromosomal copies are synthesized during the overnight growth phase in the presence of antibiotic by virtue of an appropriate resistance gene and a bacterial origin of replication engineered into the plasmid. After amplification, plasmids are purified from $E$. coli and typically introduced into an alternative host cell where the appropriate transcription and translational regulator sequences engineered into the plasmid drive the expression of the recombinant protein of interest. Mammalian Chinese hamster ovary (CHO) cells are the most common expression system for antibody-based therapeutics, and we have developed high-throughput transfection and expression methods for this cell expression system [20-22].

Plasmids are used extensively in recombinant DNA work to shuttle gene fragments between $E$. coli, where the plasmids are amplified and $\mathrm{CHO}$ cells where recombinant proteins are expressed. E. coli is commonly used to amplify plasmids of interest because the organism has a short generation time $\left(\sim 40 \mathrm{~min}\right.$ at $\left.37{ }^{\circ} \mathrm{C}\right)$, is easy to manipulate in the laboratory, and each cell can only take up one plasmid at a time. Extraction and purification of plasmids from $E$. coli involves lysing the cells, removal of bacterial cellular debris and selective recovery of the plasmid in a procedure broadly termed plasmid DNA purification. Plasmids can be purified from a wide range of overnight culture volumes. Plasmids purified from 1-2 $\mathrm{mL}$ of overnight cultures are commonly referred to as "miniprep" plasmid DNA purification. Purifying large numbers of plasmids at the miniprep scale is a tedious and ergonomically challenging endeavor. Consequently, an automated workstation for plasmid purification at small-scale will add considerable value across a wide range of biological disciplines where workflows require large numbers of plasmids to be processed.

Transfection and transient expression of recombinant proteins in CHO cells requires high-quality plasmid DNA [23-25]. Plasmids must have a super-coiled topology, as nicked or relaxed DNA (single-cut on one of the DNA strands), or linear DNA fragments (double-cut) are susceptible to rapid degradation by cellular nucleases. Preparations of plasmid DNA must be relatively free of bacterial genomic DNA and RNA to allow for accurate quantification of plasmid concentrations since protein expression is sensitive to the amount of input plasmid used for transfection of mammalian cells. Finally, plasmid preparations must be free of cytotoxic bacterial lysate and endotoxin contaminants, as well as extraction buffers since these can interfere with DNA quantification, $\mathrm{CHO}$ cell growth, or the formation of complexes between the plasmids and lipid or cationic polymers commonly used to allow passage of DNA through the cell membranes.

Historically, one of the most popular methods for plasmid isolation involves the differential precipitation of bacterial chromosomal DNA and super-coiled plasmids under alkaline conditions. In this method bacterial cells are treated with a lysozyme solution to weaken the cell walls, and then the cells lysed with sodium dodecyl sulfate (SDS) under alkaline conditions to selectively denature the chromosomal DNA while leaving the plasmid intact. Rapid neutralization of the mixture results in aggregation of chromosomal DNA, and SDS-protein complexes which can then be removed by centrifugation. Plasmid DNA is precipitated by ethanol precipitation [26]. The observation that DNA could bind to glass in the presence of chaotropic salts and then be quantitively recovered under conditions of neutral $\mathrm{pH}$ led to the use of silica membrane filters as a high-throughput alternative to ethanol precipitation [27]. Currently there are many commercially available kits for extraction of plasmid DNA from $E$. coli $[28,29]$.

Numerous high-throughput plasmid purification systems that rely on selective binding of plasmid DNA to magnetic beads, chaotropic beads or silica membrane filters have been commercialized. Automated magnetic bead-based plasmid purification systems have been shown to give equivalent plasmid yields and DNA purity as off-line procedures. How- ever, information on protein yields following transfection into mammalian cells is not available [30,31]. Billeci and co-workers describe a custom built automation station that employs specialized purification tips containing chaotropic beads to purify plasmids from E. coli [32]. The authors demonstrate equivalent plasmid yields, purity, and sequencing scores compared to off-line methods. A GFP reporter plasmid transfected in monkey kidney cells (COS7) using expensive specialized transfection reagents was used to determine the suitability of plasmids for downstream expression in mammalian cells. The authors did not report antibody titers following transfection in $\mathrm{CHO}$ cells which are more sensitive to transfection conditions and are the predominant cell line used for commercial expression of biotherapeutics. Furthermore, plasmid normalization and mixing for downstream expression in mammalian cells requires manual transfer to another system. Filter-based plasmid purification approaches generally yield higher amounts of plasmid than beadbased approaches, but generally have a throughput of only 1-8 plates [33-35]. Recently DNA yields and purity on an automated system were shown to be equivalent to off-line protocols, but there is no information of protein yields following transfection into mammalian cells [34].

Our laboratory has consistently achieved high yields of transfectiongrade plasmid DNA using a 96-well filter-based plasmid purification system (QIAprep 96 Turbo Miniprep). To avoid the use of specialized purification tips and expensive transfection reagents, we elected to continue to use the Turbo Miniprep kit for our high-throughput plasmid purification needs. To remain consistent with several other integrated platforms in our laboratory, we chose the Beckman i-series liquid handler with the Amplius Positive Pressure Devices (PPD) as the foundation of our Automated Miniprep Plasmid Station which we have termed AMPS. Successful enablement and full validation of the system depended on observing equivalent antibody yields from plasmids purified on the AMPS compared to our standard off-line protocols following transfection into $\mathrm{CHO}$ cells. Furthermore, because normalization and mixing of large numbers of LC and HC plasmids is a lengthy process, we incorporated this capability into our automated workstation.

As part of our discovery pipeline, we have enabled high-throughput cloning and mammalian transient transfection protocols to empower and accelerate the expression and screening of 1000s of antibodies against target proteins of interest. To date high-throughput isolation of plasmid DNA suitable for expression in $\mathrm{CHO}$ cells is a rate limiting step in our early-stage discovery pipeline. Here we describe a custom-designed Automated Miniprep Plasmid Station (AMPS) enabling high-throughput purification of plasmid DNA from $E$. coli for downstream expression of recombinant proteins in $\mathrm{CHO}$ cells. Plasmids are extracted and purified from $E$. coli cells using components from a commercially available kit. The station can be programed to determine plasmid concentrations based on absorbance readings at $260 \mathrm{~nm}$. These values are then used to normalize and mix plasmids encoding the antibody LC and HC sequences at optimal concentrations for downstream expression in $\mathrm{CHO}$ cells. The quality of plasmid DNA purified on the AMPS is equivalent to currently used manual protocols as determined by antibody titers following transient expression in CHO cells. AMPS can process twenty 96-deep-well plates of pelleted $E$. coli cells in a fully operator-independent manner. It executes the plasmid purification and quantification protocols from twenty 96-deep-well plates in six hours and takes another two hours to mix the cognate LC and HC plasmid pairs for subsequent downstream expression in $\mathrm{CHO}$ cells.

\section{Material and methods}

\section{Automated workstation design}

The AMPS (Fig. 1A and B) is flanked by two enclosed Biomek i7 hybrid liquid handlers (Beckman Coulter Life Sciences, Brea, CA) oriented in opposite directions each having a HEPA filter unit to provide a controlled work environment. Each liquid handler is equipped with a 96-MultiChannel head (1-1200 $\mu \mathrm{L}$ ) and a Span-8 pipettor. Integrated on 

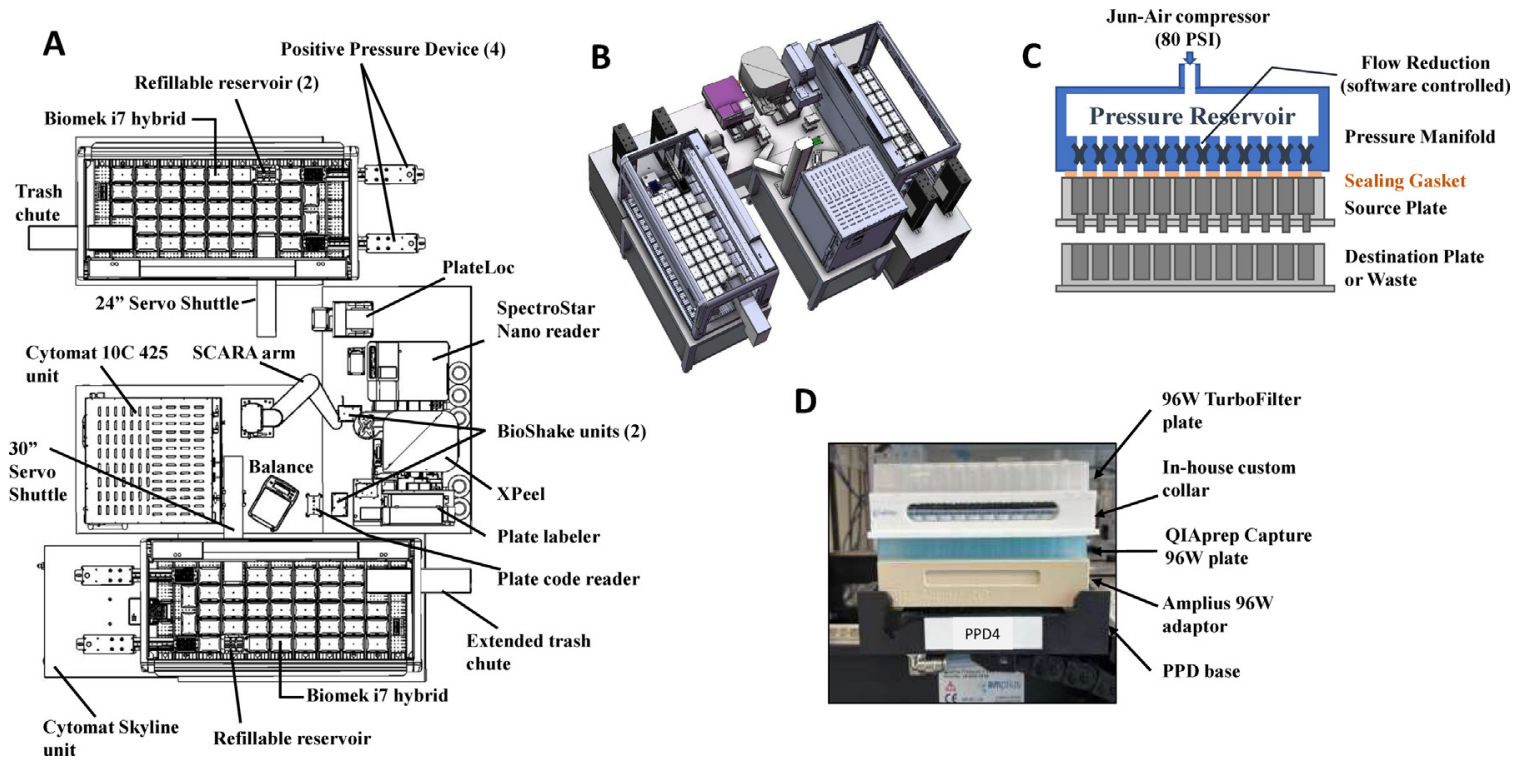

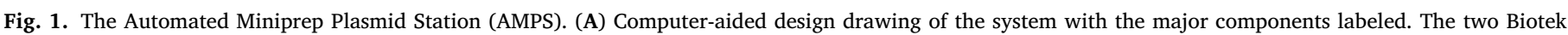

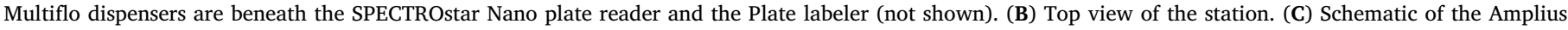

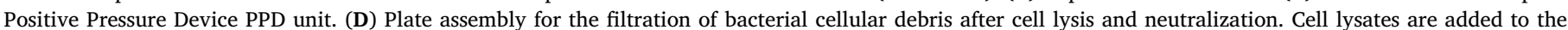

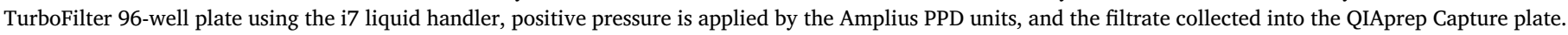

the side of each liquid handler are two fully automated Amplius Positive Pressure device (PPD) units (Amplius, Rostock, Germany) (Supplemental Fig. S1A). These consist of a 96-well pressure manifold with controlled inlets, a sealing gasket that sits on top of the source plate and a 96-well destination plate or waste drain (Fig. 1C). Two floor-level JunAir compressors (Jun-Air, Benton Harbor, MI) provide a constant highvolume air pressure to each of two PPD units. Waste material is handled by house vacuum and stored in a flammable-resistant container. Two Cytomat SkyLine (Thermo Fisher Scientific, Waltham, MA) plate storage and delivery units feed plasticware directly onto the decks of the two Biomek liquid handlers as needed (Supplemental Fig. S1B and C). On the deck of each liquid hander is a self-refilling reservoir (Amplius) for a constant supply of an ethanol-based wash solution from a pressurized flame-resistant container. At the front of the station is a Cytomat 10C-425 unit for storage of 96-deep-well blocks containing $E$. coli pellets, as well as for plates containing purified plasmid DNA. There are two Biotek Multiflo dispensers (Biotek, Winooski, VT) for dispensing resuspension, lysis, and neutralization buffers, as well as a Sartorius Entris (Sartorius, Göttingen, Germany) balance for on-the-fly liquid handling verification. In addition, the AMPS contains a SPECTROstar Nano plate reader (BMG Labtech, Ortenberg, Germany), four BioShake elm 3000 units (QInstruments, Jena, Germany), a PlateLoc sealer (Agilent, Santa Clara, CA) and a XPeel desealer (Brooks Life Sciences, Indianapolis, IN). Plates are moved around the station by a Precise PF3400 collaborative extended reach arm (Precise Automation, Fremont, CA) as well as Servo Shuttles (Beckman Coulter Life Sciences) extending from the Biomek i7 hybrids.

The instruments, plate movements and scheduling are controlled by SAMI EX software (Beckman Coulter Life Sciences). Beckman's Data Acquisition Reporting Tool (DART) links sample identification to sample data throughout the workflow. The DART database is used to calculate DNA concentrations from absorbance values provided by the SPECTROstar Nano plate reader and outputs a liquid transfer file to the Biomek i7 liquid handlers for mixing plasmids encoding the LC and HC antibody sequences at the correct concentrations for downstream transfections and optimal expression in CHO cells.

Qualification of the automated workstation

We qualified the AMPS using E. coli cells (strain DH5 $\alpha$, Zymo Research, Irvine, CA) pelleted in 96-deep-well plates and harboring plas- mids encoding either the LC or the HC of a proprietary control antibody. Beveled $2 \mathrm{~L}$ flasks containing one liter of $2 \mathrm{X}$ YT growth medium supplemented with $100 \mu \mathrm{g} / \mathrm{mL}$ carbenicillin were inoculated with a single colony of $E$. coli transformed with a proprietary DNA plasmid encoding either the HC or LC of a control antibody. Both plasmids contain the $\beta$-lactamase (bla) gene conferring resistance to carbenicillin. After incubation at $37{ }^{\circ} \mathrm{C}$ overnight $(16-18 \mathrm{~h}$ ) with shaking at $250 \mathrm{rpm}, 950 \mu \mathrm{L}$ of the overnight culture was aliquoted into each well of a 96-deep-well plate while keeping the bacteria in constant suspension to ensure equal distribution of the cells into each well. Bacteria were pelleted by centrifugation at $1460 \times \mathrm{g}$ for $10 \mathrm{~min}$. We removed the culture media by inverting the plates and blotting them on paper towels. We stored the plates at $-20{ }^{\circ} \mathrm{C}$ in an off-line unit until needed for testing on the AMPS.

Scientists loaded all the plates containing frozen bacterial pellets into the Cytomat 10C-425 units of the AMPS for processing at the same time. The bacterial pellets were fully thawed by the time they were removed from the Cytomat storage units by the Precise arm and placed on the MultiFlo dispensers. Components of the QIAprep 96 Turbo Miniprep kit (cat. \#27193; Qiagen, Valencia, CA) are used to extract and purify plasmid DNA. The two Multiflo dispensers on the station are used to add re-suspension Buffer P1 (250 $\mu \mathrm{L})$ containing RNase A and LyseBlue to the bacterial pellets, Buffer P2 to lyse the cells (250 $\mu \mathrm{L}$ ), and Buffer N3 $(350 \mu \mathrm{L})$ to neutralize the lysate and precipitate the bacterial chromosomal DNA (all buffers from QIAprep 96 Turbo Miniprep kit). The plates are transferred to the BioShake units ( $2 \mathrm{~mm}$ throw) and mixed after addition of Buffer P1 ( $5 \times \mathrm{g}$ for $180 \mathrm{~s}$ ), Buffer P2 (1 $\mathrm{x} g$ for $30 \mathrm{~s}$ ) and Buffer N3 (7 x $g$ for $10 \mathrm{~s})$.

The Biomek i7 hybrids are used to transfer $(\sim 700 \mu \mathrm{L})$ the bacterial lysates to the 96W TurboFilter plates for filtration of the bacterial cellular debris by the PPD units (Fig. 1D). The white-colored collar was 3Dprinted in-house to improve separation between the TurboFilter plate and the QIAprep Capture plate (Supplemental Fig. S2). Bacterial lysates are forced through the TurboFilter plate by the PPD units at $400 \mathrm{mbar}$ of positive pressure for $30 \mathrm{~s}$ and the filtrate collected in the QIAprep Capture plate.

We printed the collar using the MakerBot MethodX 3D printer (Dynamism, Chicago IL) and a Gen2 experimental extruder (TPLA Onyx Black spool). The Cloudprint slicer was set to $70 \%$ infill with $2 \mathrm{~mm}$ wall thickness, and a $1 \mathrm{~mm}$ removable raft using print material for support. Warping was reduced by setting the chamber temperature of $50{ }^{\circ} \mathrm{C}$ and 
extruder conditions at $210 \mathrm{c}$ at $50 \mathrm{~mm} / \mathrm{s}$. We optimized flow rates by adding small viewing ports to the prototype to allow for visual optimization. The viewing ports were removed in the final print for strength. The dimensions of the collar (Supplemental Fig. S2), and the script ( ${ }^{*}$. stl) used to print the collar is available (Supplementary information).

The grippers on the Biomek i7 hybrid units are used to discard the TurboFilter plate, and to remove the custom-printed collar. Plasmid DNA is captured on the silica membrane of the QIAprep Capture plate by forcing the cleared lysate through the plate at a pressure of 55 mbar for $60 \mathrm{~s}$. The flow through is sent to waste. The filters are washed two times with $900 \mu \mathrm{L}$ of the Buffer PE wash buffer (QIAprep 96 Turbo Miniprep kit) drawn from the self-filling reservoirs and applied to the QIAprep Capture plate by the Biomek i7 hybrid. In each case the Buffer $\mathrm{PE}$ wash buffer is allowed to sit on the filters for five minutes prior to push-through. Positive pressure of $75 \mathrm{mbar}$ for $150 \mathrm{~s}$ is used for the first wash and $75 \mathrm{mbar}$ for $60 \mathrm{~s}$ is used for the $\mathrm{s}$ wash. A final blow-through at $5000 \mathrm{mbar}$ for $180 \mathrm{~s}$ is done to remove any residual Buffer PE wash buffer prior to plasmid elution.

For the plasmid elution step, the Biomek places a 96-well microtiter collection plate at the base of the PPD units, followed by the Amplius 96-well adaptor and the QIAprep Capture plate on top. Elution Buffer (EB) (QIAprep 96 Turbo Miniprep kit) is added ( $80 \mu \mathrm{L}$ ) to the center of the silica membrane at the bottom of the QIAprep plate by the Biomek i7 liquid handlers and incubated for five minutes to hydrate the membrane prior to the addition of another $50 \mu \mathrm{L}$ of EB. Eluants are collected following application of $25 \mathrm{mbar}$ of positive pressure for $120 \mathrm{~s}$ and then 1000 mbar for four seconds.

The AMPS determined DNA absorbance measurements by transferring $8 \mu \mathrm{L}$ of plasmid eluants into $32 \mu \mathrm{L}$ of water in 96-well Half Area UVStar microplates (Greiner Bio-One, Monroe, NC) and reading the plates on the SPECTROstar Nano reader. Plasmid concentrations are calculated in DART, and the Biomek Span-8 pipettor is used to normalize and mix the LC and HC plasmids at the correct concentrations for downstream expression in $\mathrm{CHO}$ cells. The 8-Span pipettor back-fills the wells to a final volume of $15 \mu \mathrm{L}$ with water. Long-term DNA storage plates are prepared by mixing $5 \mu \mathrm{L}$ of plasmid DNA (regardless of concentration) into $20 \mu \mathrm{L}$ of TE buffer pH 7.0 buffer (\#AM9861, Invitrogen, Carlsbad, CA) into 384-well plates using the Biomek i7 multichannel head and the plates stored at $-80^{\circ} \mathrm{C}$.

The station can extract and purify plasmid DNA from twenty 96-well plates of overnight $E$. coli cultures and quantify DNA yields in about six hours, and then normalize and mix the LC and HC plasmids for downstream CHO expression in an additional two hours. After loading the Qiagen kit components and the plasticware required to execute the procedure, the system is run in a completely operator-independent manner.

\section{Off-line plasmid purification and quantification}

We purified plasmids from $E$. coli plates prepared at the same time as those used on the AMPS using the QIAprep 96 Turbo Miniprep kit in an off-line mode. Off-line Biomek liquid handlers add the re-suspension, lysis, and neutralization buffers. Plates were vortexed after adding the re-suspension buffer to the pellets and inverted gently 3-5 times after addition of the lysis and neutralization buffers, with care being taken to ensure that the addition of the neutralization buffer occurred within five minutes of cell lysis. We found that centrifugation of the plates at 2862 $\mathrm{x} g$ for ten minutes after addition of the neutralization buffer aids in the transfer of the lysates to the TurboFilter plates. Lysates were filtered, plasmids bound to the QIAprep Capture plate and filters washed with the Buffer PE wash buffer using a vacuum apparatus as suggested by the manufacturer. We removed trace amounts of wash buffer by including an additional centrifugation step (2862 $\mathrm{x} g$ for ten minutes) prior to elution of plasmids. Elution volumes (EB Buffer) and incubation times were as described on the AMPS, except we collected eluants by centrifugation (2862 $\mathrm{x} g$ for five minutes). We determined DNA absorbance values using either an off-line SPECTROstar Omega reader (BMG Labtech) or an off-line NanoDrop 8000 reader (ThermoFisher Scientific, Waltham, MA). The Qiagen Plasmid Megaprep kit (\#12181) was used for largescale purification of the same plasmid used in the 96-well experiments from a $500 \mathrm{~mL}$ overnight $E$. coli culture. Plasmids were electrophoresed on a 1\% agarose gel (\#1613044, BioRad, Hercules, CA) for $60 \mathrm{~min}$ at $100 \mathrm{~V}$ and visualized by staining with ethidium bromide.

\section{Antibody expression in $\mathrm{CHO}$ cells}

In preliminary experiments, plasmids encoding the LC (kappa constant) and HC (IgG4 constant) of the control antibody, were mixed at ratios ranging from $200 \mathrm{ng}$ to $1600 \mathrm{ng}$ (Supplemental Fig. S4) in 96deep-well Master-blocks (Greiner Bio-One) using the Lynx LM I800 VVP (Variable Volume Pipettor) dispenser (Dynamic Devices, Phoenix, AZ). When comparing antibody titers following transfections of plasmid purified on the AMPS with those off-line the LC and HC plasmids were mixed at a ratio of 600:600 ng (original protocol) or 1400:1200 ng (optimized). We transiently transfected $\mathrm{CHO}$ cells using Transfection Grade Linear Polyethylenimine Hydrochloride (PEI) with a molecular weight of 40,000 (Polysciences, Warrington, PA) essentially as described previously [20]. Six days post-transfection the plates were centrifuged to pellet the $\mathrm{CHO}$ cells and supernatants containing the expressed antibodies harvested.

\section{Quantification of antibodies}

The CHO supernatants were diluted 1:5 in PBS pH 7.4 (ThermoFisher Scientific) into $40 \mu \mathrm{L}$ final volume for each sample in a Tilted-bottom (TW384) Microplate (Sartorius). We determined antibody concentrations using Protein G (ProG) Dip and Read Biosensors (Sartorius) on the high-throughput Octet HTX bio-layer Interferometer instrument (Pall Corporation, Port Washington, NY). Sample values were fit to a human IgG4 reference curve and final antibody concentrations calculated using the Data Analysis HT (ver. 10) software embedded in the instrument.

\section{Results}

The AMPS is designed to execute the following tasks: (i) extraction and purification of plasmid DNA from E. coli pellets, (ii) quantification of plasmid yields, (iii) replica stamping of DNA plates for long-term storage $\left(-80{ }^{\circ} \mathrm{C}\right)$, and (iv) normalization and mixing of plasmids encoding antibody LC and HC sequences for expression in mammalian cells (Fig. 2). Prior to full deployment of the AMPS in our antibody discovery workflows, we sought to compare plasmids yields, DNA quantification procedures and antibody titers following transfection in $\mathrm{CHO}$ cells using plasmids purified on the AMPS to our standard off-line procedures. We used the same batch of 96-deep-well plates containing $E$. coli pellets prepared as described in the Qualification of the Automated Workstation section of the Material and Methods for each experiment comparing on-line and off-line protocols.

Super-coiled plasmid DNA is required for optimal expression of proteins in mammalian cells $[23,36]$. Initial experiments on the AMPS revealed primarily plasmids in the relaxed or nicked form and these plasmids yielded significantly lower antibody titers compared to those purified off-line following transfection in CHO cells (Supplemental Fig. S3). As a result, we optimized parameters for each step of the AMPS purification protocol as outlined in the Material and Methods section. Homogenous re-suspension of the $E$. coli pellets on the BioShake units after addition of Buffer P1 is important for maximal plasmid yields. Complete cell lysis with Buffer P2 and thorough neutralization after addition of Buffer N3 is monitored by the loss of color from the LyseBlue reagent added to Buffer P1. Conditions are carefully controlled to minimize plasmid nicking due to prolonged exposure to alkaline Buffer P2, while relatively gentle mixing on the BioShake units ensure negligible bacterial chromosomal DNA contamination in the plasmid preparations due to shearing. 


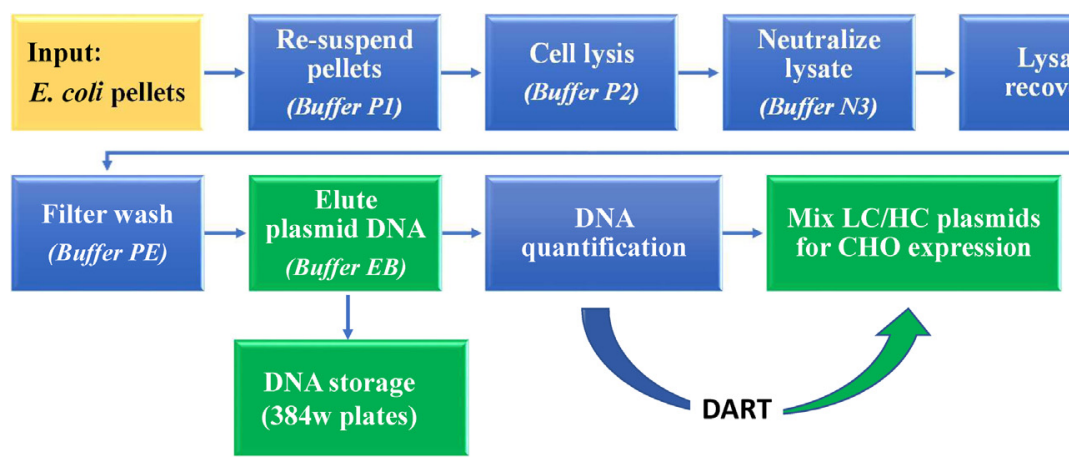

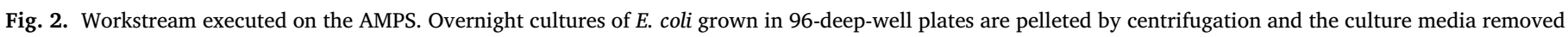

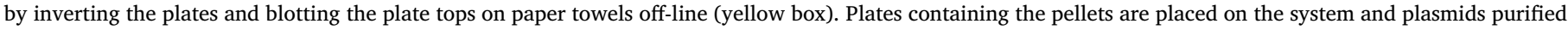

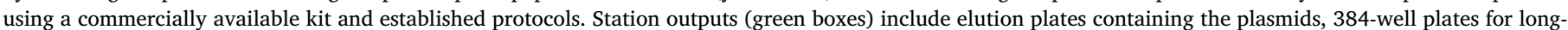

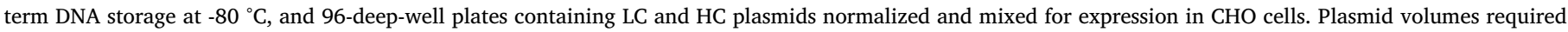

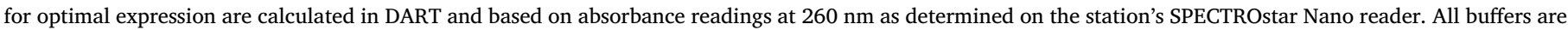
from the QIAprep 96 Turbo Miniprep kit.

Complete removal of the buffers used in the re-suspension, lysis, neutralization, and wash steps is important to ensure efficient DNA:PEI complex formation and optimal antibody expression in CHO cells. In our standard off-line protocol, the QIAprep Capture plates are centrifuged at $2862 \mathrm{x} g$ for ten minutes to ensure complete removal of residual plasmid extraction and wash buffers. We tested several strategies designed to minimize contamination of extraction and wash buffers during the plasmid elution step on the AMPS. This included (i) incubating the Buffer $\mathrm{PE}$ wash buffer with the filters for five minutes prior to push-through (PE $+5 \mathrm{~min}$ ), (ii) washing the filters two times with $1500 \mu \mathrm{L}$ (PE-1500 $\mu \mathrm{L}$ ), and (iii) implementing a third wash (3X-PE). Finally, we implemented a two-step plasmid elution protocol to maximize plasmid yields.

\section{Plasmid topology}

In mammalian cells expression of proteins encoded on extrachromosomal DNA requires plasmids to maintain a super-coiled topology. Consequently, we compared relative amounts of super-coiled DNA purified on the AMPS using the QIAprep 96 Turbo Miniprep kit to those obtained using the standard off-line protocol executed by two different operators. To this end LC (7 kbp) and HC (7.7 kbp) plasmids purified offline, or on the AMPS using a variety of wash conditions, were normalized to $500 \mathrm{ng}$, and electrophoresed on an agarose gel and visualized to compare plasmid topology (Fig. 3). As expected, commercial preparations (Aldeveron, Fargo, ND) of the control plasmid purified by chromatography (Lanes 3 and 11), or from the Qiagen Megaprep kit (Lanes 4 and 12) gave predominantly super-coiled plasmid. Significantly, similar relative amounts of super-coiled DNA were also obtained if the plasmids were purified off-line by the two operators (Lanes 5-6 and 13-14), or on the AMPS (Lanes 7 and 15). Interestingly, treatments designed to remove traces of extraction and wash buffers including PE +5 min, PE-1500 $\mu \mathrm{L}$, or $3 \mathrm{X}-\mathrm{PE}$ had minimal effects on the amounts of super-coiled plasmid relative to the nicked (relaxed) and linear forms (Lanes 8 and 16, Lanes 9 and 17, and Lanes 10 and 18 respectively). The absence of a distinct band above that of the relaxed nicked plasmid suggests no significant contamination of bacterial genomic DNA, while the absence of smearing suggests minimal RNA contamination.

\section{Plasmid purity and yields}

Plasmid yields are typically determined based on absorbance readings at an ultraviolet wavelength of $260 \mathrm{~nm}$. DNA purity is commonly assessed by determining ratios obtained from absorbance measurements taken at $260 \mathrm{~nm}$ and $230 \mathrm{~nm}$ (OD260/OD230), or $260 \mathrm{~nm}$ and $280 \mathrm{~nm}$ (OD260/OD280). For DNA, an OD260/OD280 ratio of 1.8-1.9 is optimal. A ratio of $<1.7$ suggests protein contamination, while a value $>$
2.0 suggests remnants of the basic solution used to lyse the cells $[37,38]$. In contrast, a OD260/OD230 ratio of 2.0-2.2 suggests plasmid preparations that are free of substantial contamination with organic compounds such as guanidine $\mathrm{HCl} /$ guanidine (QIAprep kits), phenol or the Trizol reagent. To determine the yields and purity of plasmids produced on the AMPS, we measured absorbance values at wavelengths of 230, 260 and $280 \mathrm{~nm}$ off-line using the NanoDrop 8000 reader.

The OD260/OD280 ratios of all LC and HC plasmids purified on the AMPS were consistently within the acceptable range (1.8-1.9), regardless of the elution treatment and furthermore were similar to those obtained by two different operators using the standard off-line protocol (Table 1). In the case of the LC plasmid, the off-line miniprep protocol, as well as a five minute incubation in Buffer PE wash buffer (PE+5 $\min$ ), or a $1500 \mu \mathrm{L} \mathrm{PE}$ wash (PE-1500) all returned plasmid preparations with OD260/OD230 ratios within the acceptable range (2.0-2.2). In contrast, LC plasmids purified on the AMPS without modification of the wash protocol (AMPS), or with an additional wash (3X-PE) gave OD260/OD230 ratios significantly less than 2.0. In the case of the HC plasmid, the OD260/OD230 ratios for plasmids purified off-line varied from 1.9-2.1, while plasmids purified on the AMPS were all 2.1 regardless of wash buffer treatment. Significantly, the well-to-well variation in OD260/OD230 ratios was lowest with the PE +5 min treatment relative to other procedures on the AMPS; particularly in the case of the LC plasmid.

Plasmid DNA yields obtained by both operators using the standard off-line protocol were similar and averaged $219 \mu \mathrm{g} / \mathrm{mL}$ for the LC and $210 \mu \mathrm{g} / \mathrm{mL}$ for the HC plasmid. Plasmid concentrations for the LC chain were 10-20\% less on the AMPS relative to those off-line. In contrast, except for the PE- $1500 \mu \mathrm{L}$ treatment, DNA concentrations for the HC plasmid were higher $35-45 \%$ higher on the AMPS station. The OD260/OD280 and OD260/OD230 ratios for the HC plasmid on the AMPS were within the recommended range suggesting that contamination with RNA or organic-based purification buffers do not explain the elevated readings at $260 \mathrm{~nm}$. In the case of both the LC and the HC plasmids, DNA concentrations of individual wells were slightly greater when plasmids were purified on the AMPS than off-line.

In the off-line protocol, residual Buffer PE wash buffer is removed from the QIAprep Capture plates by centrifugation and blotting of the nozzles on absorbance paper, while on the Amplius PPD units, a final blow-out at 5000 mbar for 180 seconds is used to remove residual buffer. Despite using the same total volume of Elution Buffer $(130 \mu \mathrm{L})$, the offline protocol typically returned $100 \mu \mathrm{L}$ of final elution volume, while the AMPS station returned 80-90 $\mu \mathrm{L}$. In the case of the LC plasmid, the total yields for the AMPS station were $30-40 \%$ lower than the average of the two off-line operators, while the yields of the HC plasmids were generally similar. 


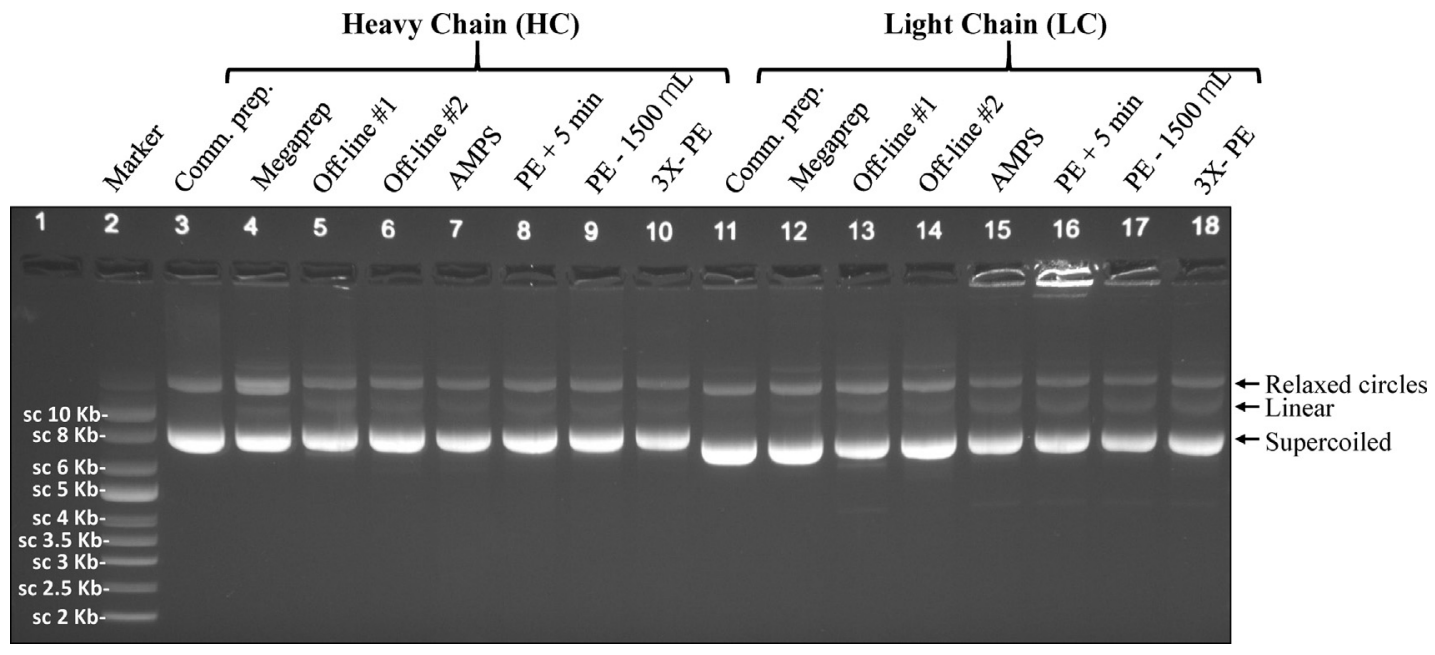

Fig. 3. Topology of plasmid DNA extracted and purified on the AMPS. Plasmid DNA ( $500 \mathrm{ng}$ ) was electrophoresed on a $1 \%$ agarose gel. Marker DNA is in Lane 2, and interpretation of the different plasmid topologies indicated on the far right (sc denotes super-coiled marker DNA). Treatments are as follows: plasmid DNA purified by chromatography by an external commercial enterprise (Lanes 3 and 11), DNA purified using the Qiagen Plasmid Megaprep kit (Lanes 4 and 12), DNA purified off-line by Operators \#1 and \#2 (Lanes 5 and 6 and 13 and 14), plasmids purified on the AMPS (Lanes 7 and 15), DNA purified on the AMPS with the added treatment of allowing the PE wash buffer to contact the membrane on the QIAprep Capture plates for 5 min prior to push-through (Lanes 8 and 16), washing the membranes with $1500 \mu \mathrm{l}$ of buffer (Lanes 9 and 17), or introduction of a third Buffer PE wash (Lanes 10 and 18). Plasmids encoding the HC and LC chains of the control antibody are indicated. Expected size of the HC super-coiled plasmid is 7.7 kilo base pairs, and that of the LC 7.0 kilo base pairs.

Table 1

Absorbance ratios and yields of plasmids purified on the AMPS.

\begin{tabular}{|c|c|c|c|c|c|c|c|c|c|c|c|}
\hline \multicolumn{12}{|c|}{ LC plasmid } \\
\hline & & \multicolumn{2}{|c|}{ OD260/OD280 } & \multicolumn{2}{|c|}{ OD260/OD230 } & \multicolumn{3}{|c|}{ Concentration $(\mu \mathrm{g} / \mathrm{mL})$} & \multicolumn{3}{|c|}{ Total yield $(\mu \mathrm{g})$} \\
\hline & & Average & SD & Average & SD & Average & SD & Relative to $\mathrm{O} 1+\mathrm{O2}^{\mathrm{d}}$ & Average & SD & Relative to $\mathrm{O} 1+\mathrm{O} 2$ \\
\hline & Off-line Op. \# $1^{\text {a }}$ & 1.9 & 0.01 & 2.1 & 0.02 & 234.0 & 14.8 & 1.0 & 28.1 & 1.8 & 1.0 \\
\hline & Off-line Op. \# $2^{\mathrm{b}}$ & 1.9 & 0.01 & 2.1 & 0.03 & 205.1 & 13.2 & & 24.6 & 1.6 & \\
\hline \multirow[t]{4}{*}{ AMPS } & AMPSc & 1.8 & 0.16 & 1.7 & 0.44 & 201.2 & 32.7 & 0.92 & 18.1 & 2.9 & 0.69 \\
\hline & $\mathrm{PE}+5 \mathrm{~min}$ & 1.9 & 0.03 & 2.0 & 0.12 & 198.9 & 39.6 & 0.91 & 17.9 & 3.6 & 0.68 \\
\hline & PE - $1500 \mu l$ & 1.9 & 0.06 & 2.0 & 0.22 & 178.8 & 63.1 & 0.82 & 16.1 & 5.7 & 0.61 \\
\hline & 3X-PE & 1.9 & 0.11 & 1.8 & 0.35 & 187.5 & 42.6 & 0.85 & 16.9 & 3.8 & 0.64 \\
\hline \multicolumn{12}{|c|}{ HC plasmid } \\
\hline & & \multicolumn{2}{|c|}{ OD260/OD280 } & \multicolumn{2}{|c|}{ OD260/OD230 } & \multicolumn{3}{|c|}{ Concentration $(\mu \mathrm{g} / \mathrm{mL})$} & \multicolumn{3}{|c|}{ Total yield $(\mu \mathrm{g})$} \\
\hline & & Average & SD & Average & SD & Average & SD & Relative to $01+\mathrm{O2}^{\mathrm{d}}$ & Average & SD & Relative to $01+02$ \\
\hline \multirow{6}{*}{ AMPS } & Off-line Op. $\# 1^{\text {a }}$ & 1.9 & 0.01 & 2.1 & 0.15 & 216.2 & 15.3 & 1.0 & 25.9 & 1.8 & 1.0 \\
\hline & Off-line Op. $\# 2^{\mathrm{b}}$ & 1.9 & 0.02 & 1.9 & 0.25 & 203.9 & 16.9 & & 24.9 & 2.0 & \\
\hline & AMPSc & 1.9 & 0.01 & 2.1 & 0.06 & 305.4 & 54.8 & 1.45 & 27.5 & 4.9 & 1.08 \\
\hline & $\mathrm{PE}+5 \mathrm{~min}$ & 1.9 & 0.02 & 2.1 & 0.03 & 283.1 & 44.0 & 1.34 & 25.5 & 4.0 & 1.00 \\
\hline & PE - $1500 \mu 1$ & 1.9 & 0.01 & 2.1 & 0.07 & 239.9 & 11.2 & 1.14 & 21.6 & 1.0 & 0.85 \\
\hline & 3X-PE & 1.9 & 0.01 & 2.1 & 0.02 & 304.7 & 32.5 & 1.45 & 27.4 & 2.8 & 1.08 \\
\hline
\end{tabular}

a Off-line values from operator \#1 (Op.) represent the averages from 8 wells.

b Off-line values from operator \#2 (Op.) represent the averages from 24 wells.

${ }^{c}$ Each of the treatments on the AMPS represent the averages from 8 wells.

${ }^{\mathrm{d}}$ DNA concentrations and total yields from the two operators (Op.) were averaged and used as a reference for DNA yields of plasmids purified on the AMPS station.

In summary, the AMPS with a five minute incubation of the Buffer $\mathrm{PE}$ wash buffer prior to push-through (PE $+5 \mathrm{~min}$ ) consistently provided plasmid preparations that were free of RNA and organic buffer contaminants, and at adequate concentrations and yields to perform multiple small-scale CHO cell transfections in support of early-stage discovery screening assays.

\section{Antibody titers}

Maximal transient expression of proteins from extra-chromosomal plasmid DNA in CHO cells requires that the plasmid be super-coiled, that it can be accurately quantified, and that the preparation is free of contaminants that can interfere with efficient PEI:DNA complex formation. We confirmed that the station could provide sufficiently high-quality plasmid DNA to allow for protein expression in $\mathrm{CHO}$ cells prior to full integration of the AMPS into our discovery workflows. Consequently, we compared control antibody titers following $\mathrm{CHO}$ transfection with LC and HC plasmids purified on the AMPS to those provided by two operators using the standard off-line protocol (Table 2). Antibody titers were compared at a LC and HC plasmid ratio of 600:600 ng (original protocol), as well as at a ratio of 1400:1200 ng (optimized), since in a matrix-type experiment we demonstrated that higher amounts of plasmid DNA resulted in higher antibody production while still economizing the amounts of HC plasmid needed (Supplemental Fig. 4S).

The average titers of the control antibody from the two operators were as expected $(190 \mu \mathrm{g} / \mathrm{mL})$ following transfection of CHO cells in a 96-well plate format with $600 \mathrm{ng}$ each of the LC and HC plasmids purified off-line. An increase in DNA transfection amounts to $1400 \mathrm{ng}: 1200$ ng (LC:HC) resulted in a 2.5-fold increase in antibody titers. At both DNA concentrations, plasmids purified on the AMPS with a five minute 
Table 2

Antibody titers from plasmids purified on the AMPS.

\begin{tabular}{|c|c|c|c|c|}
\hline \multicolumn{5}{|c|}{ Antibody Titers (LC:HC plasmid ratio of $600 \mathrm{ng}: 600 \mathrm{ng})^{\mathrm{a}}$} \\
\hline & & \multicolumn{2}{|c|}{ Antibody Concentrations ( $\mu \mathrm{g} / \mathrm{mL})$} & \multirow[t]{2}{*}{ Yields relative to $(\mathrm{O} 1+\mathrm{O} 2)$} \\
\hline & & Average & SD & \\
\hline \multirow{6}{*}{ AMPS } & Off-line Operator 1 & 166.8 & 12.8 & \multirow[t]{2}{*}{$1.00^{c}$} \\
\hline & Off-line Operator $2^{\mathrm{b}}$ & 211.8 & 20.2 & \\
\hline & AMPS & 165.6 & 59.5 & 0.87 \\
\hline & $\mathrm{PE}+5 \mathrm{~min}$ & 177.5 & 34.2 & 0.94 \\
\hline & $\mathrm{PE}-1500 \mu \mathrm{l}$ & 140.8 & 52.7 & 0.74 \\
\hline & $3 \mathrm{X}-\mathrm{PE}$ & 147.2 & 25.4 & 0.78 \\
\hline
\end{tabular}

Antibody Titers (LC:HC plasmid ratio of $1400 \mathrm{ng}: 1200 \mathrm{ng}$ )

\begin{tabular}{|c|c|c|c|c|}
\hline & & \multicolumn{2}{|c|}{ Antibody Concentrations $(\mu \mathrm{g} / \mathrm{mL})$} & \multirow[t]{2}{*}{ Yields relative to $(\mathrm{O} 1+\mathrm{O} 2)$} \\
\hline & & Average & $\mathrm{SD}$ & \\
\hline \multirow{6}{*}{ AMPS } & Off-line Operator \#1 & 490.3 & 29.0 & \multirow[t]{2}{*}{1.00} \\
\hline & Off-line Operator \#2 & 597.6 & 33.1 & \\
\hline & AMPS & 487.9 & 154.0 & 0.90 \\
\hline & $\mathrm{PE}+5 \mathrm{~min}$ & 521.8 & 81.6 & 0.96 \\
\hline & $\mathrm{PE}-1500 \mu \mathrm{l}$ & 410.3 & 138.8 & 0.75 \\
\hline & 3X-PE & 444.0 & 62.8 & 0.82 \\
\hline
\end{tabular}

a Antibodies in the CHO supernatants were quantified 6 days post-transfection.

b Off-line values represent an average of 24 wells. Values reported for all treatments on the AMPS are an average of 8 wells.

c Antibody concentrations from plasmids purified off-line by the two operators are averaged and used as a reference for antibody titers yielded by plasmids purified on the AMPS.

incubation of the Buffer PE wash on the QIAprep Capture plates prior to push-through ( $\mathrm{PE}+5 \mathrm{~min}$ ) yielded the highest antibody titers on the AMPS. Importantly, these titers were equivalent to those obtained from plasmids purified off-line. Although a third wash with the Buffer PE wash buffer (3X-PE) resulted in slightly less well-to-well variability in antibody titers within a plate relative to the $\mathrm{PE}+5$ min treatment, average antibody yields following a third wash were significantly lower than those obtained off-line.

\section{DNA quantification on the AMPS}

Maximal antibody titers following transient transfection of $\mathrm{CHO}$ cells in a 96-well plate format are highly dependent on accurate quantification of plasmid DNA and correct LC:HC plasmid ratios (Supplemental Fig. S4). After purification on the AMPS, plasmids are diluted 5-fold in water and the absorbance at $260 \mathrm{~nm}$ measured on-line on the SPECTROstar Nano reader. The DART database is configured to calculate the DNA concentration and generate a LC:HC plasmid normalization and mix file for subsequent transfection and expression in $\mathrm{CHO}$ cells. The Span-8 pipettor on the Biomek i7 liquid handler is used to mix the two plasmids at the correct concentrations and to back fill each well with water to a final volume of $15 \mu \mathrm{L}$.

To confirm the accuracy of the 5-fold plasmid dilution step on the Biomek i7 liquid handlers prior to obtaining the absorbance measurements, we purified LC and HC plasmid DNA from five 96-well plates each using the AMPS. Plasmid concentrations from Column 1 of all ten plates were determined using an off-line NanoDrop 8000 reader. We then diluted all the plasmid samples in the ten 96-well plates 5-fold on the AMPS using the Biomek i7 MultiChannel heads and read the same 80 samples from Column 1 again on the NanoDrop 8000 reader. The mean difference between the observed plasmid concentration after the 5 -fold dilution and the expected concentration was $-1.18 \mu \mathrm{g} / \mathrm{mL}$ with a $95 \%$ confidence interval of -1.17 to -1.19 (Fig. 4A). The corresponding standard deviation of the differences was 1.97 (or $4.8 \%$ RSD) with a Pearson correlation value of 0.97 , thus confirming the accuracy of the plasmid dilution step by the Biomek i7 liquid handler on the AMPS.

After 5-fold dilution, the plasmid concentrations of all ten plates were determined by the SPECTROstar Nano reader on the AMPS. The NanoDrop 8000 reader was used to confirm plasmid concentrations for samples in Column 10 across all ten plates. The average concentration of the diluted samples on the SPECTROstar Nano reader was $38.8 \mu \mathrm{g} / \mathrm{mL}$, while that of the NanoDrop 8000 reader was $36.3 \mu \mathrm{g} / \mathrm{mL}$. The DNA concentrations determined by the SPECTROstar Nano reader and the NanoDrop 8000 reader had a mean difference of $-2.49 \mu \mathrm{g} / \mathrm{mL}$ with a $95 \%$ confidence interval of -0.16 to 0.16 (Fig. 4B). The corresponding standard deviation of the differences was 2.86 (or $7.4 \%$ RSD) with a Pearson correlation value of 0.92 . The close correlation between the two readers validated the correct calibration and functionality of the SPECTROstar Nano reader on the AMPS.

\section{Consistency of plasmid yields across a twenty plate run}

To demonstrate the uniformity of plasmid purification within a plate and between plates, we processed twenty 96-deep-well plates on the AMPS. LC or HC plasmids were purified from ten 96-deep-well plates each, diluted 5-fold, their absorbance measured at $260 \mathrm{~nm}$, and plasmid concentrations calculated in DART. All 20 plates were processed in a completely operator-independent manner. We observed no mechanical issues on the AMPS during the eight hour run.

The average LC plasmid DNA concentration for each of the ten plates $(\mathrm{n}=96)$ ranged from $129.0-138.6 \mu \mathrm{g} / \mathrm{mL}$, with standard deviations (SD) ranging from 9.8-13.1, and percent relative standard deviation (\%RSD) ranging from $7.5 \%-9.8 \%$ (Table 3 ). On a per plate basis, average elution volumes ranged from $86 \mu \mathrm{L}-89 \mu \mathrm{L}$, yielding $11.2 \mu \mathrm{g}-12.1 \mu \mathrm{g}$ of DNA/well. Across the ten plates $(\mathrm{n}=960)$, the average LC plasmid concentration was $132.5 \mu \mathrm{g} / \mathrm{mL}$, $(\mathrm{SD}=12.1, \% \mathrm{RSD}=9.1)$, with an average plasmid yield of $11.6 \mu \mathrm{g}$ /well. In the case of the HC plasmids, the average concentration for each plate $(\mathrm{n}=96)$ ranged from $135.2-151.0$ $\mu \mathrm{g} / \mathrm{mL}$, with standard deviations (SD) ranging from 11.9- 22.7, and percent relative standard deviation (\%RSD) ranging from $8.2 \%-15.3 \%$. Average elution volumes ranged from $82 \mu \mathrm{L}-84 \mu \mathrm{L}$, yielding 11.1-12.5 $\mu \mathrm{g}$ total DNA/well. Across the ten HC plasmid plates $(n=960)$, the average plasmid concentration was $142.3 \mu \mathrm{g} / \mathrm{mL},(\mathrm{SD}=16.0, \% \mathrm{RSD}=11.2)$, with an average total plasmid yield of $11.8 \mu \mathrm{g} /$ well. The reason for the slightly higher HC plasmid yields and greater variation in concentrations relative to that of the LC plasmid might be due to inherent differences in plasmid size, or the presence of the glutamine synthetase gene on the LC plasmid. In addition, slight differences in the performance of the PPD units have been observed when the units are run simultaneously. Slight 
A

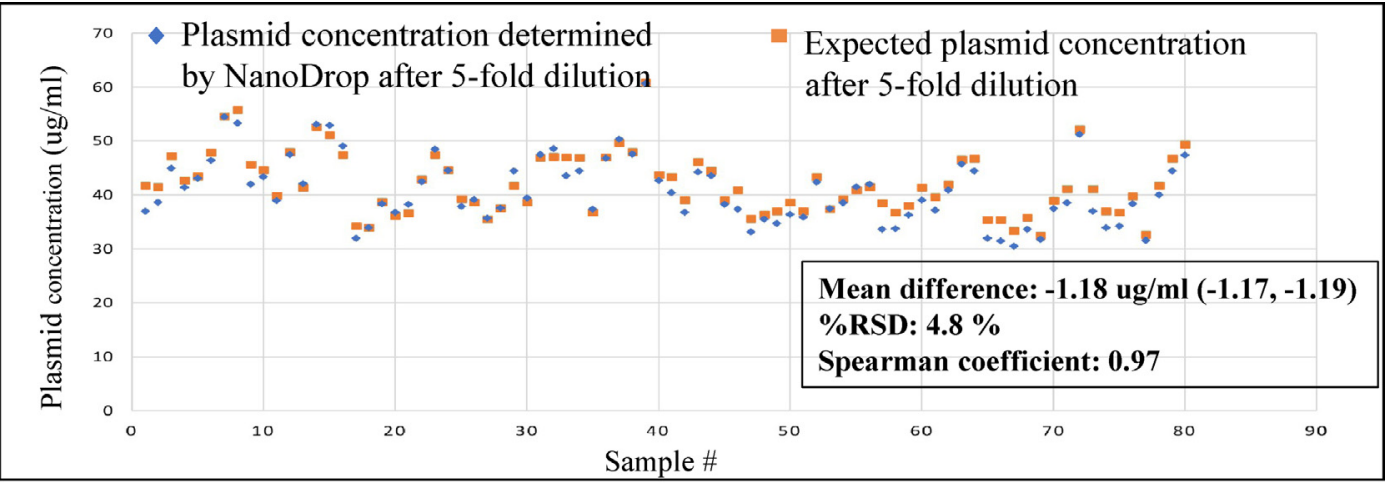

B

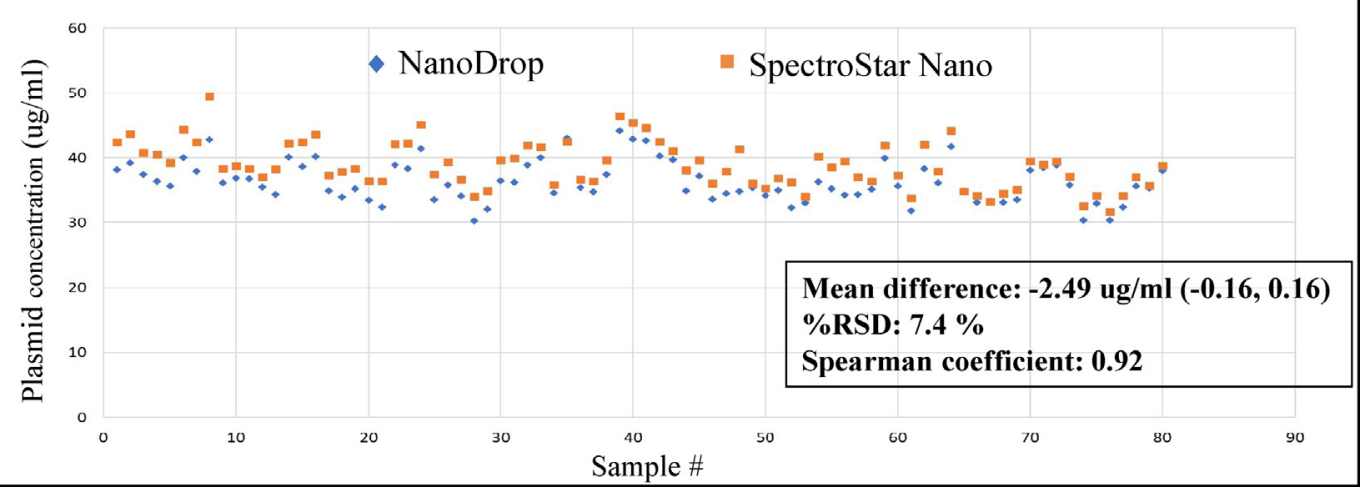

Fig. 4. Dilution and quantification of plasmid DNA on the AMPS. (A) Plasmids encoding the LC and HC sequences of a control antibody were purified from ten 96-well plates, and DNA concentrations of samples from Column 1 of the 96-well microtiter plates determined off-line using the NanoDrop 8000 reader (80 samples). Plasmids were diluted 5-fold on the AMPS and samples from the same column measured on the off-line NanoDrop 8000 reader again. Measured plasmid yields following a 5-fold dilution were compared to expected yields (B) Plasmid concentrations after a 5-fold dilution were compared from absorbance readings taken on the SPECTROstar Nano reader on the AMPS or using the off-line NanoDrop 8000 reader for samples in Column 10 across the ten plates.

Table 3

Plasmid DNA purified on the AMPS from twenty 96-well plates of $E$. coli pellets.

\begin{tabular}{|c|c|c|c|c|c|c|c|c|c|}
\hline \multirow[b]{2}{*}{ 96-well plate } & \multicolumn{3}{|c|}{$\begin{array}{l}\text { Light Chain plasmid concentration } \\
(\mu \mathrm{g} / \mathrm{mL})\end{array}$} & \multirow[t]{2}{*}{$\begin{array}{l}\text { DNA yield/well } \\
(\mu \mathrm{g})^{\mathrm{a}}\end{array}$} & \multirow[b]{2}{*}{ 96-well plate } & \multicolumn{3}{|c|}{$\begin{array}{l}\text { Heavy Chain plasmid concentration } \\
(\mu \mathrm{g} / \mathrm{mL})\end{array}$} & \multirow[t]{2}{*}{$\begin{array}{l}\text { DNA yield/well } \\
(\mu \mathrm{g})\end{array}$} \\
\hline & Average & SD & $\%$ RSD & & & Average & SD & $\%$ RSD & \\
\hline $\begin{array}{l}\text { LC }^{b} \text { - Plate } 1 \\
(n=96)\end{array}$ & 129.0 & 10.4 & $8.0 \%$ & 11.5 & $\begin{array}{l}\text { HC - Plate } 1 \\
(n=96)\end{array}$ & 143.9 & 12.9 & $8.9 \%$ & 12.2 \\
\hline LC - Plate 2 & 129.0 & 9.8 & $7.6 \%$ & 11.5 & HC - Plate 2 & 135.2 & 13.2 & $9.7 \%$ & 11.5 \\
\hline LC - Plate 3 & 126.7 & 11.1 & $8.7 \%$ & 11.3 & HC - Plate 3 & 144.8 & 11.9 & $8.2 \%$ & 12.0 \\
\hline LC - Plate 4 & 137.0 & 11.2 & $8.2 \%$ & 12.1 & HC - Plate 4 & 136.1 & 15.6 & $11.4 \%$ & 11.1 \\
\hline LC - Plate 5 & 130.3 & 12.0 & $9.2 \%$ & 11.3 & HC - Plate 5 & 148.7 & 22.7 & $15.3 \%$ & 12.2 \\
\hline LC - Plate 6 & 134.0 & 13.1 & $9.8 \%$ & 11.6 & HC - Plate 6 & 138.3 & 14.1 & $10.2 \%$ & 11.4 \\
\hline LC - Plate 7 & 134.3 & 13.1 & $9.7 \%$ & 11.8 & HC - Plate 7 & 136.5 & 12.9 & $9.5 \%$ & 11.1 \\
\hline LC - Plate 8 & 138.6 & 11.8 & $8.5 \%$ & 12.1 & HC - Plate 8 & 148.8 & 13.3 & $8.9 \%$ & 12.2 \\
\hline LC - Plate 9 & 137.0 & 10.2 & $7.5 \%$ & 11.8 & HC - Plate 9 & 139.2 & 15.4 & $11.0 \%$ & 11.4 \\
\hline LC - Plate 10 & 129.3 & 11.6 & $9.0 \%$ & 11.2 & HC - Plate 10 & 151.0 & 15.2 & $10.1 \%$ & 12.5 \\
\hline $\begin{array}{l}\text { LC plasmid } \\
\text { average } \\
(n=960)\end{array}$ & 132.5 & 12.1 & $9.1 \%$ & 11.6 & $\begin{array}{l}\text { HC plasmid } \\
\text { average } \\
(n=960)\end{array}$ & 142.3 & 16.0 & $11.2 \%$ & 11.8 \\
\hline
\end{tabular}

a Elution volumes are estimated by weighing each plate before and after elution.

b Plasmid encoding the light chain (LC) and heavy chain (HC) of the control antibody.

differences in the performance of the PPD units would be particularly relevant during the elution step.

In the case of both the LC and HC plasmids, the AMPS provided consistent antibody titers within a 96-well plate, as well as between the ten plates containing the same plasmid (LC or HC). Maximal antibody titers following transfection of $\mathrm{CHO}$ cells in a 96-well plate format requires up to $1.4 \mu \mathrm{g}$ of the LC plasmid and $1.2 \mu \mathrm{g}$ of the HC plasmid. The AMPS provides sufficient plasmid yields and at adequate concentrations for multiple CHO transfections, enabling multiple high-throughput antibody screening campaigns for both binding and function.

\section{Discussion}

Here we present the successful design, qualification, and enablement of a fully integrated plasmid DNA miniprep station capable of purifying plasmids from twenty 96-deep-well plates of $E$. coli pellets, quantifying the DNA, and preparing plates for downstream expression in $\mathrm{CHO}$ cells. All these operations can be completed in a fully operator-independent manner. Plasmid concentrations and total yields on the AMPS are similar to those obtained off-line using the same commercially available kits and manufacturer's suggested protocols. Plasmid preparations are free 
of significant bacterial chromosomal DNA and cellular RNA, as well as the buffers used in the plasmid purification process. Most importantly, to ensure sufficient material for downstream screening assays, plasmids purified and mixed for $\mathrm{CHO}$ expression on the AMPS provide similar antibody titers to those obtained using plasmids purified off-line.

Plasmids are used in many aspects of recombinant DNA work including expression of recombinant proteins, biotherapeutic antibody discovery and engineering efforts, genome engineering, and protein mode-ofaction studies. Plasmids containing DNA fragments of interest are typically amplified in bacteria such as E. coli and subsequent purification is a relatively labor and time intensive endeavor; particularly when $1000 \mathrm{~s}$ of plasmids must be processed. To our knowledge this is the first report of a fully integrated plasmid purification workstation using a 96-well plate filter-based approach that can purify, quantify, and mix $\mathrm{CHO}$ cell expression-grade plasmid miniprep DNA at a throughput of twenty 96well plates in a fully operator-independent manner.

The AMPS bridges upstream high-throughput molecular biology procedures and downstream $\mathrm{CHO}$ expression protocols. Numerous commercially available high-throughput colony pickers can be used to pick $E$. coli colonies from plates and inoculate liquid media in 96-deep-well plates for overnight growth and subsequent plasmid purification on the AMPS. The system is also aligned with downstream high-throughput mammalian transfection protocols [22]. The plasmid purification protocols described herein are applicable across a wide range of biological research disciplines where large plasmid banks are used including antibody discovery, protein engineering processes, biological mode of action investigations, ORFeome studies and CRISPR/Cas screens.

We made four critical modifications to the QIAprep 96 Turbo Miniprep protocol to allow for the processing of large numbers of 96well plates on the AMPS. The manufacturer's protocol recommends gently mixing the plates by inversion after addition of the lysis buffer (Buffer P2) and then again after addition of the neutralization buffer (Buffer N3), with care being taken to ensure that lysates are neutralized within five minutes of the lysis step. This procedure ensures minimal shearing of bacterial chromosomal DNA which can co-purify with plasmid DNA, and in addition prevents conversion of super-coiled plasmids to the nicked or relaxed form by alkaline hydrolysis. Mixing plates by inversion is one step of the process where we wanted to explore alternative options that would be more straightforward to automate. Consequently, in a first example of a protocol modification on the AMPS, thorough cell lysis and neutralization were performed by gentle addition of buffers using the Biotek Multiflo dispensers, and careful calibration of shaking conditions on the BioShake units. We configured the scheduling software to ensure that the lysis step was strictly limited to four minutes per plate. Two additional examples of modifications from the off-line protocol include using the Amplius PPD units instead of centrifugation to filter the neutralized lysate, as well as a high pressure blow-through step on the PPD units in place of centrifugation and blotting to remove the residual Buffer PE wash buffer. Finally, in the off-line protocol eluants are recovered by centrifugation, while the Amplius PPD units are used on the AMPS. Despite differences from the manufacturer's suggested off-line protocol, careful calibration of the Biomek i7 liquid handlers, BioShake units and the Amplius PPD units on the AMPS allowed for purification of plasmids that were $>90 \%$ super-coiled, had low to undetectable levels of cellular DNA and RNA, were free of extraction buffers, and most importantly resulted in equivalent antibody titers.

The manufacturers introduced the Amplius PPD version 3 units and the Cytomat SkyLine automated plate storage and delivery systems to the market in the last two years and both instruments proved critical to the success of the AMPS. The Amplius PPD units were used instead of centrifugation or vacuum-applied pressure during the filtration, wash, and elution steps of the plasmid purification protocol. The Amplius PPD units provide the opportunity to perform automated Solid Phase Extraction; a widely used procedure for separating analytes from complex solutions. The device applies positive pressure to each well of a 96-well microtiter plate independently allowing for even liquid flow across all wells of a plate regardless of whether some wells are empty, clogged, or contain different volumes of liquid. The pressure applied to each well from the reservoir above the plate can be carefully controlled allowing for precise rates of liquid flow. The device can be fully integrated with the Biomek i7 liquid handlers, providing rapid and facile execution of fully automated workflows.

Processing twenty 96-well plates of $E$. coli pellets through plasmid purification, DNA quantification, and preparation of CHO transfection plates requires forty 96-well microtiter plates (for elution and DNA quantification), ten 96-deep-well plates for $\mathrm{CHO}$ expression, twenty Turbofilter and QIAprep Capture plates each, five 384-well plates for longterm DNA storage, as well as P200 $(n=21)$ and P50 $(n=40)$ tip boxes (Beckman Coulter Life Sciences). The Cytomat SkyLine storage unit contains fourteen stackers and can house up to 728 microtiter plates, or any combination of different plate and tip types. In addition, the units can also house Turbofilter and QIAprep Capture plates. The high capacity and versatility of the Skyline storage unit is achieved by stacking plasticware of the same type directly on top of each other in the rack. A plate lift within the presentation tower (Supplemental Fig. S1B) provides rapid access to plasticware housed in the stacks in a sequential manner. The presentation tower of the Skyline storage unit is centered in an open cavity between the two Amplius PPD devices on each half of the AMPS and includes a Transfer Station on top of the unit which removes the plasticware from the lift and presents it for access by the gripper on the adjacent Biomek i7 liquid handler. The Skyline storage units can also store plates containing purified plasmids and 96-deep-well CHO transfection plates upon completion of the run. The large capacity of the Skyline storage units, the versatility of their stackers, and the ability to accept processed plates helped reduce the size of the AMPS.

In our hands, the QIAprep 96 Turbo Miniprep kit consistently provides predominantly super-coiled plasmids at high yields. Consequently, we designed the AMPS to use these plates and directly compared off-line and on-line purification procedures to enable and validate the AMPS. We anticipate that with minor modifications in pressure and timing, the AMPS could be used to purify plasmids from $E$. coli pellets using 96-well filter-based kits from other commercial sources as well.

The AMPS takes about six hours to purify and quantify plasmids from twenty 96-well plates, and another two hours to normalize and transfer LC and HC plasmids to a separate set of plates that can be stored on the system until transfected into $\mathrm{CHO}$ cells on a different workstation. In contrast, off-line 96-well plasmid purification protocols are tedious and typically take skilled operators $2-3$ days to complete. The AMPS throughput is well aligned to the current high-throughput antibody discovery processes executed by scientists in the Automation Group and provides them with the opportunity to work on other projects. The AMPS could also be used to process samples collected from multiple scientists throughout our facility, or even as a substitute for mid-scale plasmid purification $(100-900 \mu \mathrm{g})$ of replicate samples in a 96-well plate.

\section{Funding statement}

MC, DER, ML, PWA, JC, FJT, and TC are, or were at the time of the work described here, employees and stockholders of Eli Lilly \& Company. Eli Lilly \& Company provided support in the form of salaries for the authors and research funding. Eli Lilly \& Company approved the publication of this work. The employment of the authors by Eli Lilly \& Company does not alter our adherence to SLAS Technology policies on data sharing and materials.

\section{Acknowledgements}

The authors would like to thank Candyd Velasquez for transfection of the CHO cells, Suntara Cahya for assistance with the statistical analysis and John M Beals for critical reading of the manuscript. We thank Randy Whitmire, Wayne Schmidt, Tim Sherrill, Amy Gibson, Brandon 
Corbin, and Stephan Meister from Beckman Coulter Life Sciences for invaluable discussions around the design of the station, build-out of the station and its enablement. We acknowledge other members of the Biologics Automation and High-Throughput Technologies group for additional technical support and helpful discussions.

\section{Supplementary materials}

Supplementary material associated with this article can be found, in the online version, at doi:10.1016/j.slast.2022.01.005.

\section{References}

[1] Kaplon H, Reichert JM. Antibodies to watch in 2019. MAbs 2019;11:219-38.

[2] Lu R, Hwang Y, Liu I, et al. Development of therapeutic antibodies for the treatment of diseases. J Biomed Sci 2020;27:1-30.

[3] Moving up with the monoclonals https://biopharmadealmakers.nature.com/users/ 9880-biopharma-dealmakers/posts/53687-moving-up-with-the-monoclonals (accessed Aug 2, 2021).

[4] Dunn-Walters D, Townsend C, Sinclair E, et al. Immunoglobulin gene analysis as a tool for investigating human immune responses. Immunol Rev 2018;284:132-47.

[5] Shi B, Ma L, He X, et al. Comparative analysis of human and mouse immunoglobulin variable heavy regions from IMGT/LIGM-DB with IMGT/HighV-QUEST. Theor Biol Med Model 2014;11:1-11.

[6] Lefranc MP, Lefranc G. Immunoglobulins or antibodies: IMGT® bridging genes, structures and functions. Biomedicines 2020;8:319.

[7] Jarasch A, Koll H, Regula JT, et al. Developability assessment during the selection of novel therapeutic antibodies. J Pharm Sci 2015;104:1885-98.

[8] Kennedy PJ, Oliveira C, Granja PL, et al. Monoclonal antibodies: technologies for early discovery and engineering. Crit Rev Biotechnol 2018;38:394-408.

[9] Harris KE, Aldred SF, Davison LM, et al. Sequence-based discovery demonstrates that fixed light chain human transgenic rats produce a diverse repertoire of antigen-specific antibodies. Front Immunol 2018;9:889-900.

[10] Corti D, Misasi J, Mulangu S, et al. Protective monotherapy against lethal ebola virus infection by a potently neutralizing antibody. Science 2016;351:1339-42.

[11] Chiu ML, Goulet DR, Teplyakov A, et al. Antibody structure and function: the basis for engineering therapeutics. Antibodies 2019;8:55-135.

[12] Haraya K, Tachibana T, Igawa T. Improvement of pharmacokinetic properties of therapeutic antibodies by antibody engineering. Drug Metab Pharmacokinet 2019;34:25-41.

[13] Wang Q, Chung CY, Chough S, et al. Antibody glycoengineering strategies in mammalian cells. Biotechnol Bioeng 2018;115:1378-93.

[14] Azizi A, Lam W, Phenix H, et al. No training required: experimental tests support homology-based DNA assembly as a best practice in synthetic biology. J Biol Eng 2015;9:8-13.
[15] Celie PHN, Parret AHA, Perrakis A. Recombinant cloning strategies for protein expression. Curr Opin Struct Biol 2016;38:145-54.

[16] Gibson DG. Enzymatic assembly of overlapping DNA fragments. Methods Enzymol 2011;498:349-61.

[17] Irwin CR, Farmer A, Willer DO, et al. In-Fusion ${ }^{\circledR}$ cloning with vaccinia virus DNA Polymerase. Methods Mol Biol 2012;890:23-35.

[18] Marillonnet S, Grützner R. Synthetic DNA assembly using golden gate cloning and the hierarchical modular cloning pipeline. Curr Protoc Mol Biol 2020;130:1-33.

[19] Weber E, Engler C, Gruetzner R, et al. A modular cloning system for standardized assembly of multigene constructs. PLoS One 2011;6:e16765.

[20] Rajendra Y, Hougland MD, Alam R, et al. A high cell density transient transfection system for therapeutic protein expression based on a CHO GS-Knockout cell line: process development and product quality assessment. Biotechnol Bioeng 2015;112:977-86.

[21] Crissman J, Lin Y, Separa K, et al. RNase H-dependent PCR enables highly specific amplification of antibody variable domains from single B-Cells. PLoS One 2020;15:1-21.

[22] Jones BE, Brown-Augsburger PL, Corbett KS, et al. The neutralizing antibody, LYCoV555, protects against SARS-CoV-2 infection in nonhuman primates. Sci Transl Med 2021;13:1-17.

[23] Carbone A, Fioretti FM, Fucci L, et al. High efficiency method to obtain supercoiled DNA with a commercial plasmid purification kit. Acta Biochim Pol 2012;59:275-8.

[24] Maucksch C, Bohla A, Hoffmann F, et al. Transgene expression of transfected supercoiled plasmid DNA concatemers in mammalian cells. J Gene Med 2009;11:444-53.

[25] Von Groll A, Levin Y, Barbosa MC, et al. Linear DNA low efficiency transfection by liposome can be improved by the use of cationic lipid as charge neutralizer. Biotechnol Prog 2006;22:1220-4.

[26] Birnboim HC, Doly J. A rapid alkaline extraction procedure for screening recombinant plasmid DNA. Nucleic Acids Res 1979;7:1513-23.

[27] Vogelstein B, Gillespie D. Preparative and analytical purification of DNA from Agarose. Proc. Natl. Acad. Sci. U. S. A. 1979;76:615-19.

[28] BioCompare. No title https://www.biocompare.com/Nucleic-Acid-Purification/ 25857-Plasmid-DNA-Purification-Kits/ (accessed Aug 2, 2021).

[29] Qiagen. QIAprep miniprep handbook. QIAprep Miniprep Handb. 2012, 1-44.

[30] Hamilton. Automation of the Zyppy TM -96 plasmid MagBead miniprep; 2017.

[31] ThermoScientific. Automated nucleic acid extraction workstation; 2012.

[32] Billeci K, Suh C, Di Ioia T, et al. Implementation of an automated high-throughput plasmid DNA production pipeline. J Lab Autom 2016;21:765-78.

[33] Hudson Robotics. DNA, RNA, protein extraction and purification; 2016.

[34] Hamilton. NucleoSpin ${ }^{\circledR} 96$ plasmid transfection-grade. 2019, 4-5.

[35] Scherling, C.; Group, T.; Antar, S.; et al. Positive pressure-based plasmid preparation using QIAprep ${ }^{\circledR}$ chemistries. 2020.

[36] Stadler J, Lemmens R, Nyhammar T. Plasmid DNA purification. J Gene Med 2004;6:54-66.

[37] ThermoScientificNanoDrop: assessment of nucleic acid purity. Protoc Prod Manuals 2011:1-2.

[38] Wilfinger WW, Mackey K, Chomczynski P. Effect of PH and ionic strength on the spectrophotometric assessment of nucleic acid purity. Biotechniques 1997;22:474-81. 